\title{
Patients with schizophrenia valued self empowerment, understanding how self related to illness, and coping with relapse
}

Williams CC, Collins AA. Defining new frameworks for psychosocial intervention. Psychiatry 1999 Spring;62:61-78.

QUESTION: How do patients with schizophrenia experience their sense of self in relation to their illness?

\section{Design}

Grounded theory.

\section{Setting}

Psychiatric centre in Toronto, Ontario, Canada.

\section{Patients}

15 outpatients between 21 and 47 years of age (mean age $30 \mathrm{y}, 87 \%$ men) with schizophrenia (Diagnostic and Statistical Manual of Mental Disorders III, revised criteria) and $\geq 1$ of delusions, hallucinations, bizarre behaviour, or severe thought disorder. Patients were grouped by duration of illness: having recovered from their first psychotic episode that occurred within the previous 2 years (first episode), having had between 2 and 10 years since their first psychotic episode and $>1$ psychotic episode since the first episode (2-10 y group), and having had $>10$ years since their first psychotic episode and $>1$ psychotic episode since the first episode ( $>10$ y group).

\section{Methods}

Patients participated in a semistructured life history interview. Interviews lasted $\leq 2$ hours and were audiotaped and transcribed verbatim. Data were categorised, coded, and analysed for emergent themes by the 2 investigators.

\section{Main findings}

3 main themes emerged (fig). The first theme, the crisis created by losing control in the context of illness, referred to the patients' struggles to maintain and regain personal power and efficacy as they faced the illness and consequent difficulties with interpersonal relationships and competence. Patients' psychological and social functioning were challenged by stress and symptoms of the illness, pressures created by perceived social expectations, concerns about the ability to connect with other people, and discrepancies between the self they wished to be and the self they actually realised. The second theme focused on the process of putting it in perspective. Patients redefined their illness in terms that preserved feelings of self efficacy and stability, and that enabled them to continue pursuing personal goals. This process differed by duration of illness.

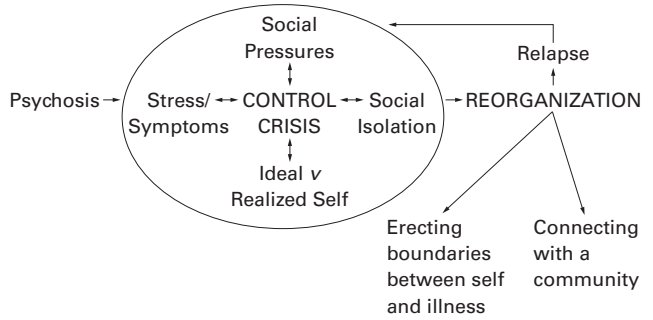

Subjective theory of illness and recovery. Reprinted with permission from Williams CC, Collins AA. Defining new frameworks for psychosocial intervention. Psychiatry 1999;62:73.
Patients in the first episode group had difficulty separating thoughts and behaviours attributed to the illness from their core beliefs and emotions and had anxiety about identifying with the community of people with mental illnesses. The $2-10$ year and $>10$ year groups clarified the boundaries between self and illness and also found a connection with a group that gave a sense of community and belonging. The third theme, coping with relapse, pertained to the unpredictability of illness and the possibility of recurring symptoms. Patients coped with future uncertainty using behavioural distractions, stress management, spirituality, and connection with an accepting social group.

\section{Conclusions}

Patients with schizophrenia struggled to maintain personal power. Patients engaged in internal and external reorganisation as they clarified boundaries between self and illness. They achieved control by increasing social support, enhancing self image, and achieving positive social connection despite persisting symptoms and the possibility of relapse.

\section{COMMENTARY}

Williams and Collins provide a thought provoking insight into people's personal experiences of schizophrenia, from which they have constructed a theoretical framework that offers new scope for psychosocial interventions. The authors chose a qualitative methodology using life history interviews with 15 people and accessed a complex web of beliefs and concerns about the illness. The sex bias of this study (13 of the 15 participants were men) is the only substantial methodological limitation.

A strength of this study is the acknowledgment of the wisdom and expertise of the patient, an issue that, with some notable exceptions, ${ }^{1}$ is often neglected within mental health research. Understanding the patient's perspective is vital in improving patient satisfaction with care and for service and professional development. ${ }^{2}$ The authors' finding that it is important to help people to identify areas in which they can play an active part in recovery confirms what many clinicians already believe is best in practice.

This paper also draws various interesting analogies between the role of hope and recovery in schizophrenia and that in other chronic illnesses, such as cancer. It challenges the nursing profession to consider the commonalities and differences between the experience and treatment of mental and physical illness, and to explore new psychosocial ways of treatment for people with schizophrenia.

Helen Lester, MB, BCH, MRCGP General Practitioner and Research Fellow Department of General Practice, Medical School University of Birmingham, Birmingham, UK

1 Rogers A, Pilgrim D. Pulling down churches: accounting for the British mental health users movement. Sociol Health Illn 1991;13:129-48.

2 Wilson J. Acknowledging the expertise of patients and their organisations. BMJ 1999;319:771-4.

3 Becker T, Thornicroft G. Community care and management of schizophrenia. Current Opinion in Psychiatry 1998;11:49-54. 